\title{
Boundary-induced orientation of dynamic filament networks and vesicle agglomerations
}

\author{
Philip Greulich ${ }^{1,2}$ and Ludger Santen ${ }^{2}$ \\ ${ }^{1}$ SUPA, School of Physics and Astronomy, University of Edinburgh, Edinburgh, UK \\ ${ }^{2}$ Fachrichtung Theoretische Physik, Universität des Saarlandes, Saarbrücken, Germany
}

(Dated: November 7, 2011)

\begin{abstract}
We find a statistical mechanism that can adjust orientations of intracellular filaments to cell geometry in absence of organizing centers. The effect is based on random and isotropic filament (de-)polymerization dynamics and is independent of filament interactions and explicit regulation. It can be understood by an analogy to electrostatics and appears to be induced by the confining boundaries; for periodic boundary conditions no orientational bias emerges. Including active transport of particles, the model reproduces experimental observations of vesicle accumulations in transected axons.
\end{abstract}

In living cells, cargo like nutrients, proteins or organelles, has to be carried to distinct locations in the cell. Many of these objects are transported by motor proteins (kinesin, dynein and myosin) that bind cargo and move along polarized intracellular filaments (microtubules, actin filaments) in a directed manner [1]. The direction of movement is determined by the polarity of the filament (plus- and a minus-end) and the motor protein species.

For particular cell functions, accumulations of cargo and concentration gradients are needed at distinct locations of the cell. For that purpose the arrangement of filaments, which determines the distribution of cargo, has to be organized properly. In some cases filaments are organized by auxiliary components to form globally polarized structures (e.g. the microtubule organizing center (MTOC) [2]). Next to these highly regulated mechanisms, motor driven transport processes take place also on less organized networks. In this article we consider a scenario where the initially ordered network of microtubules $(M T \mathrm{~s})$ in a neuronal axon is destroyed after transection of the axon. Experimental observations show that after transection, vesicles agglomerate adjusting to the geometry of the cut axon $[3,4]$. This vesicle agglomeration is important for the formation of a growth cone that is needed to recover neuronal connections. It was supposed that a re-organization of the microtubule network creates effective kinetic traps for the vesicles.

Mechanisms for self-organization of intracellular filaments were proposed in previous works, relying on extrinsic chemical gradients induced by external signals $[5,6]$, or spontaneous structure formation driven by the effective interactions of filaments mediated by molecular motors $[7-13]$.

Here, we use a complementary approach: We ask the question how structures self-organize without relying on explicit regulation mechanisms (e.g. by pre-established chemical gradients) or interactions, and discuss if they are necessary to explain the alignment of filament orientations and subsequent vesicle agglomerations in finite volumes. We consider explicitly a simple homogeneous and isotropic stochastic model for filament growth dy- namics that neglects filament-filament interactions [15]. The model considers basic filament nucleation and (de-) polymerization dynamics as considered in previous models (e.g. [17]), without an a priori preferred direction or position of filaments nor any gradients. However, the network and particle dynamics are assumed to be confined to a given geometry corresponding to the finite cell volume. We show that the confining boundary conditions in fact induce an orientation of the filament ensemble. We also present a linear theory for filament network evolution which is analogous to electrostatics and able to explain the observed orientation of filaments.

Our model is defined analogue to [15]. We apply stochastic dynamics in continuous space for filament and particle dynamics. The model rules capture the basics of filament nucleation and (de-)polymerization dynamics [1]: Filaments are nucleated with spatially homogeneous rate $\omega_{n} \rho_{m o n} \rho_{n u c}$ and isotropic random orientation. Here the values $\rho_{\text {mon }}$ and $\rho_{\text {nuc }}$ denote the (conserved) quantity of monomers and nucleation seeds in the cytosol. Filaments can grow by including segments of length $d_{s}$ at their plus-end with rate $\omega_{g} \rho_{m o n}$ and shrink by dissociating a segment at the minus- end with rate $\omega_{s}$. After nucleation, the minus-end remains capped for some time such that depolymerization at the minus-end is not possible. This restriction is removed with uncapping rate $\omega_{u}$ (which is non-zero only for actin filaments).

Particles are implemented as hard-core spheres with radius $r_{p}$ exhibiting mutual steric exclusion. Detached particles perform a continuous random walk in space. If they are within the binding range of a filament $d_{b}$, they can attach to the filament (rate $\omega_{a}$ ) and perform directed motion until they detach with rate $\omega_{d}$. We consider two species of particles: plus-particles move towards the plusend of the filament with rate $p$ per segment, while minusparticles move to the minus ends. The model dynamics are illustrated in Fig. 1. For a more detailed definition we refer to the supplemental material [14] and [15].

We want to apply the model to the experimental setup studied in [3]: Healthy axons usually contain bundles of axially orientated microtubules with plus ends pointing towards the synapse. In the experiments, axons were 
(a)

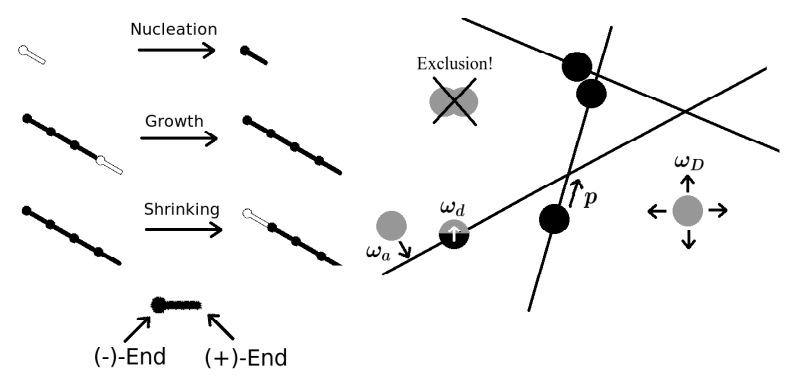

FIG. 1. Illustration of model dynamics. (a) Filaments are implemented as sequences of linear segments. These are polarized, with a plus-end where segments are created to elongate, and a minus-end where segments dissociate causing shrinking. (b) Dark gray discs are detached particles, black discs represent particles attached to filaments.

(a)

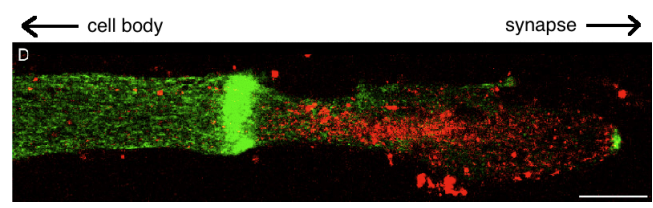

(b)

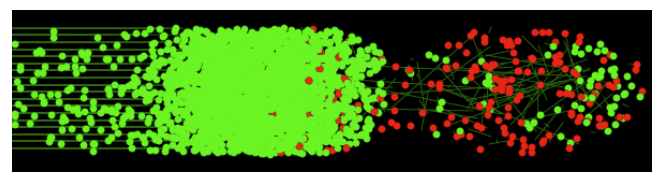

FIG. 2. (Color) Comparison between experimental results of Erez et al. [3] (a) and model results (b). The same colorcoding is used: anterograde (plus-) particles are green, retrograde (minus-) particles are red. The shape of the model axon is based on the experiment (bar $=15 \mu \mathrm{m}$ ). The deviations from the cylindrical shape have only little influence on the particle distribution, hence the accumulation is not result of a (diffusive) bottleneck (see supplemental material [16]).

transected leading to dissolution of microtubules near the location of the cut, due to calcium influx (right part of Fig. 2(a)). In that region, microtubules subsequently reassemble randomly in absence of an organizing center. Non-destroyed MTs remain and can grow again. One observes an accumulation of plus- (anterograde) vesicles at the tip and at some distance from the tip as well as minus- (retrograde) vesicles between these locations.

In the model we implement this scenario by introducing a confining volume that is based on the cylindrical shape of an axon. We use an idealized cylindrical shape as well as a shape similar to the contour of the axon used in the experiment of Erez et al. [3]. The finite volume leads to a straightforward modification of the filament dynamics: Filaments are not allowed to grow or nucleate outside the volume (see Fig. 2(b)). Starting from paral-
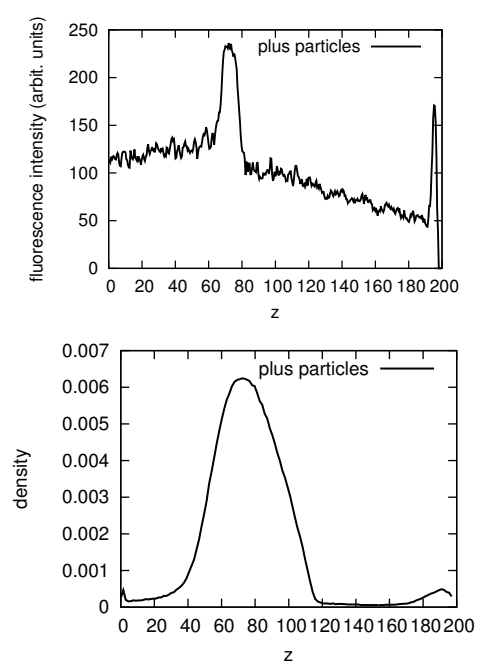

FIG. 3. Comparison between experimental [3](top) and simulated particle distribution (bottom). $\mathrm{z}=$ cylinder axis coordinate.

lel bundles of microtubules, they get dissolved (transection) within some distance from the tip (right half of the figure) and new filaments reassemble randomly without preferred direction, according to the rules defined above. Moreover, we assume that filament growth is inhibited by the steric hard-core particles such that filaments are not allowed to grow through a particle. In addition, we implement microtubule catastrophe-rescue dynamics following the model in [17]. At the left boundary, plus particles enter the system with rate $\alpha_{1}$ where also minus particles exit the system with rate $\beta_{2}$. At the other boundaries (representing the membrane), plus particles exit (exocytosis) with rate $\beta_{1}$ and minus particles enter the system (endocytosis) with rate $\alpha_{2}$.

In Fig. 2(b) a particle configuration 24000 time steps after transection (corresponding to about $4 \mathrm{~min}$ in real time) is displayed. We used the biologically motivated parameters as discussed in the supplemental material [14]. Starting with parallel filaments, they are dissolved in the right part of the system after 7000 time steps, whereupon random nucleation and growth of filaments, according to the dynamics discussed above, takes place in this region.

The comparison between experimental and model results shows that the model is able to reproduce the structure of the plus particle traps as well in the middle of the system and at the tip, but it underestimates its strength (Fig. 3(b)).

In order to understand the emergence of particle accumulations, we develop a linear theory for the network structure. The orientations of filament segments will be denoted by a unit vector $\mathbf{d}$ describing the direction of the plus-end. We quantify the structure of the network by a 
vector field

$$
\mathbf{F}(\mathbf{x}):=\left\langle\lim _{v \rightarrow 0} \frac{1}{v} \sum_{\text {fil. in } \mathrm{v}} \mathbf{d}(\mathbf{x})\right\rangle=\rho_{s}\langle\mathbf{d}(\mathbf{x})\rangle
$$

which we call filament field. Here, $v$ is a volume containing the point $\mathbf{x}$. The sum includes all filament segments in $v, \rho_{s}$ denotes the density of filament segments at the point $\mathbf{x}$ and $\langle\cdots\rangle$ denotes the ensemble average. The direction of $\mathbf{F}$ represents the average orientation of filaments, whereas its amplitude is determined by the density of filaments. $\mathbf{F}(\mathbf{x})$ hence results from a linear superposition of filament orientations. A similar formalism has been considered in [18], where it was shown numerically that the finite nucleation volume can lead to a non-zero average bias. However, in that work filament nucleating granules played the role of MTOCs. We want to understand and quantify the boundary-induced effects explicitly in absence of MTOCs by using an analytical Green's function formalism. Due to superposition, the filament field at the point $\mathbf{x}$ can be expressed by a Green's function as

$$
\mathbf{F}(\mathbf{x})=\int_{V} \mathbf{G}\left(\mathbf{x}-\mathbf{x}^{\prime}\right) \rho_{n}\left(\mathbf{x}^{\prime}\right) d x^{\prime 3}
$$

where $\mathbf{G}\left(\mathbf{x}-\mathbf{x}^{\prime}\right)$ is the field if nucleation of filaments would only be possible in a single point $\mathbf{x}^{\prime}$, and $\rho_{n}(\mathbf{x})$ denotes the relative nucleation rate. We consider the dynamics to be spatially homogeneous within the volume $V$ where filaments can nucleate, hence $\rho_{n}(\mathbf{x})=1$ within $V$ and $\rho_{n}(\mathbf{x})=0$ outside [19]. However, (2) can also be applied, when nucleation dynamics vary spatially.

Since the orientation of filaments always points away from the nucleation point, the Green's function $\mathbf{G}(\mathbf{x})$, which considers only nucleation in the origin, must have a radial structure $\mathbf{G}(\mathbf{x})=G(\mathbf{x}) \mathbf{e}_{r}$. In fact, $G(\mathbf{x})$ is the probability that a filament is within a volume unit $d x^{3}$ around $\mathbf{x}$. Since filaments are not correlated, this can be expressed by the probability $P(\mathbf{x})$ that the filament is directed towards $\mathbf{x}$ and, assuming ergodicity, the average time $\tau(\mathbf{x})$ the filament is present in $d x^{3}$ : $G(\mathbf{x})=P(\mathbf{x}) \tau(r)$. Because a filament grows isotropically in arbitrary direction, the probability that it passes the volume $d x^{3}$ located at distance $r=|\mathbf{x}|$ from the origin is given by $P(\mathbf{x})=P(r) \propto 1 / r^{2}$. In the stationary state, the net polymerization rate $\omega_{g} \rho_{\text {mon }}$ must equal the depolymerization rate $\omega_{s}$ due to conservation of monomers. If we neglect shrinking at the plus end, a filament can only disappear at a given point by minus-end depolymerization. The average filament length $l$ is independent of the distance $r$ and hence the time the filament is present at $\mathbf{x}$ is $\tau=l / \omega_{s} d_{s}$ which is also independent of $r$.

After all, the overall value of the filament field is

$$
\mathbf{G}(r) \propto \frac{\mathbf{e}_{r}}{r^{2}} .
$$

This form corresponds to the Green's function of electrostatics (Coulomb law). As a result, the filament field

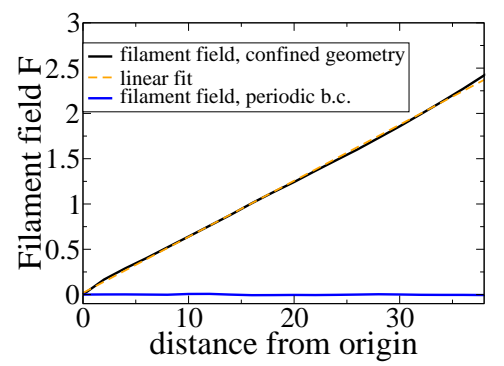

FIG. 4. (Color online) Radial component of the filament field $F(r)$ for default parameters in 3D. For pbc (blue/grey line) there is no bias in the network structure as expected due to translational symmetry. For confining sbc (black line), one observes approximately a linear behavior as predicted by the analytical theory.

inside a volume $V$ with homogeneous nucleation rate corresponds to an electrostatic field of a homogeneous charge distribution within $V$.

In order to test these results quantitatively, we consider more simple boundary conditions than in the previous example. Specifically we apply spherical boundary conditions (sbc), where the dynamic filaments are confined to a sphere of radius $R$. For comparison we also consider periodic boundary conditions (pbc).

Due to translational symmetry for pbc and non-biased dynamics there is supposed to be no overall bias and the field $\mathbf{F}$ vanishes. This is reproduced by simulations as can be seen in Fig. 4. However, if the filaments are confined to a sphere, the theory predicts that $\mathbf{F}$ has the same form as an electrostatic field inside a homogeneously charged sphere. For that geometry, Gauss' divergence theorem yields

$$
\mathbf{F}(\mathbf{x}) \sim r \mathbf{e}_{r} \quad \text { for } r<R .
$$

This linear behavior of $\mathbf{F}$, with average radial orientation of filament plus ends towards boundaries, is indeed reproduced by simulations of filament ensembles (Fig. 4).

Particles follow the emerging radial bias of the filaments leading to a separation of particle species (Fig. 5). Therefore, in the case of spherical boundary conditions, plus-particles accumulate at the boundaries while minusparticles are depleted at the boundaries and have a slight tendency towards the center. Analogue to electrostatics we conclude that, for an arbitrary geometry, random nucleation dynamics leads, in general, to an average bias of plus ends towards the boundaries.

However, for MT dynamics the Green's function needs some modifications. Since there is a chance for plus-end depolymerization, while minus ends are fixed, the Green's function has a finite range scale, given by the mean filament length. For larger distances, the effect of a nucleating filament is 'screened'. This means that the bias effect is only strong within a distance from the boundary that corresponds to the average filament length. Nonetheless, the direction of the bias remains unaffected by the 
(a)

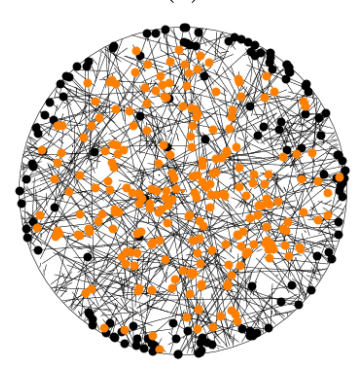

(b)

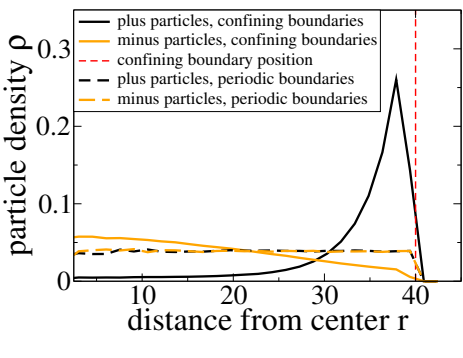

FIG. 5. (Color online) Distributions of plus- (black) and minus-particles (orange/light grey) for spherical boundary conditions.(a) Snapshot of particle configurations (Slice plane). (b) Radial density distribution of vesicles.

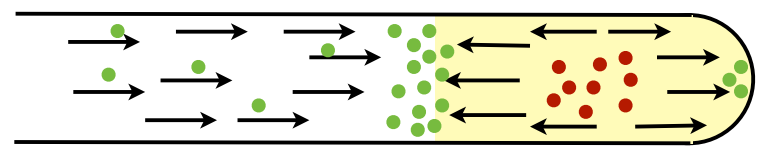

FIG. 6. (Color) Illustration of the re-organization of the microtubule network after transection. After dissolution of filaments, they re-assemble randomly in the right part (yellow region). According to our theory, plus ends of MTs (black arrows) are pointed towards the boundaries of this region. Adapted from [3].

screening. For our model geometry, the mean filament length $\left(80 r_{p}\right)$ is larger than the cylinder radius $\left(40 r_{p}\right)$, such that screening effects can be neglected.

Within this theory, we can also explain the observed distribution of particles in case of a transected axon: After dissolution of MTs, random nucleation and growth induces a bias of plus ends outwards of the nucleating region, arranging (schematically) as in Fig. 6 (only right part (yellow) is nucleating region). Together with the surviving microtubules at the left end, pointing to the right, this yields a trap for plus particles. In addition, a trap at the right tip emerges due to MTs pointing to the right. Our model, however, underestimates the strength of the particle traps compared to the experiments (Figs. 2 and 3). This discrepancy supports the interpretation given in [3] that interactions between filaments (which we have neglected) indeed increase orienta- tional correlations to enhance structural inhomogeneities in the filament networks [20] that stabilize and amplify the particle trapping. This amplification is due instabilities driven by positive feedback mechanisms, which result from filament-filament interactions $[10,13]$ and transport of nucleation promoting agents [7]. However, these theories are not able to elucidate how the initial bias emerges. Our model, in contrast, predicts a boundaryinduced bias, which can trigger an interaction-driven selforganization of the filaments towards the observed patterns.

To summarize, we studied a model for active transport on filament networks that evolve by isotropic and spatially homogeneous stochastic filament dynamics. We showed that if filament dynamics is confined within a finite volume, the filaments become oriented following the geometry of the system, despite the absence of filamentfilament interactions and chemical gradients. Applied to the geometry of a transected axon, this random dynamics is sufficient to explain the structure of experimentally observed vesicle traps, which is essential for axonal regeneration.

We developed a linear theory which is analogous to electrostatics and describes correctly the alignment of filament orientations. This theory is assumed to be generic for arbitrary geometries and predicts a preferred orientation of filament plus-ends towards boundaries. Hence we have shown that in confined geometries, concentration gradients can generally be induced by randomly generated networks of polarized filaments, even without interaction-driven self-organization or external gradients.

Our results indicate that the geometry of the cell may have strong influence on the development of the structure of the filament network: Although our model results do not reproduce the strength of vesicle traps, their structure is well described. Therefore we conclude that the mechanism presented in this work may select the emerging structures of interacting filament systems $[7,9,10,21]$. This assumption is also supported by experiments providing evidence that the cell shape may also control the organization of the cytoskeleton [22].

We want to thank M.E. Spira, Davide Marenduzzo and Marc Neef for fruitful discussions and Maren Westkott for image analysis. We also thank DFG Grants GK1276/1 and GZ SA864/3-1 for financial support. P.G. is postdoctoral fellow of DAAD.
[1] B. Alberts et al., Molecular Biology of the Cell (Garland, 2002).

[2] K. W. Wolf and K. J. Böhm, Biologie in unserer Zeit 27.2, 87 (1997).

[3] H. Erez et al., The Journal of Cell Biology 176, 497 (2007).

[4] D. Kamber, H. Erez, and M. E. Spira, Experimental neu- rology 219, 112 (2009).

[5] I. V. Maly and G. G. Borisy, Proc. Natl. Acad. Sci. USA 98, 11324 (2001).

[6] T. E. Schaus, E. W. Taylor, and G. G. Borisy, Proc. Natl. Ac. Sci. USA 104, 7086 (2007).

[7] K. Doubrovinski and K. Kruse, Phys. Rev. Lett. 99, 228104 (2007). 
[8] K. Kruse and F. Jülicher, Phys. Rev. E 67, 051913 (2003).

[9] K. Kruse et al., Phys. Rev. Lett. 92, 078101 (2004).

[10] J. A. Aström, P. B. S. Kumar, and M. Karttunen, Soft Matter 5, 2869 (2009).

[11] R. Hawkins, O. Bénichou, M. Piel, and R. Voituriez, Phys. Rev. E 80, 040903 (2009).

[12] A. Zemel and A. Mogilner, Progress of Theoretical Physics Supplement 173, 17 (2008).

[13] A. Zemel and A. Mogilner, Physical chemistry chemical physics 11, 4821 (2009).

[14] See Supplemental Material at [URL will be inserted by publisher] for detailed model dynamics and specification of parameters.

[15] P. Greulich and L. Santen, Eur. Phys. J. E 32, 191 (2010).
[16] See Supplemental Material at [URL will be inserted by publisher] for the time evolution in a cylindrical geometry without tapering.

[17] P. Hinow, V. Rezania, and J. A. Tuszyński, Phys. Rev. E 80, 31904 (2009).

[18] E. N. Cytrynbaum, V. Rodionov, and A. Mogilner, Bull. Math. Biol. 68, 1053 (2006).

[19] This normalization is chosen to separate the influence of dynamics (in $\mathbf{G}\left(\mathbf{x}-\mathbf{x}^{\prime}\right.$ ) ) and geometry (in $\rho_{n}(\mathbf{x})$ ).

[20] K. Kruse et al., Eur. Phys. J. E 16, 5 (2005).

[21] H. Y. Lee and M. Kardar, Phys. Rev. E 64, 056113 (2001).

[22] C. R. Terenna et al., Current biology 18, 1748 (2008). 